\title{
Large Right Atrial Myxoma- An Uncommon Cardiac Tumor Needs Urgent Surgery MA QUASHEM ${ }^{\mathrm{a}}$, HRIDAY RANJAN ROY ${ }^{\mathrm{b}}$, MG KIBRIA $^{\mathrm{c}}$, AEM MAZHARUL ISLAM $^{\mathrm{d}}$, AA SHAFI MAJUMDER $^{\mathrm{e}}$, NA KAMRUL AHSAN ${ }^{\mathrm{f}}$, NU AHMED ${ }^{\mathrm{g}}$
}

\author{
Summary: \\ A middle aged man was admitted into National Institute of \\ Cardiovascular Diseases with the complaints of \\ intermittent syncopal attack, dyspnoea, low grade fever \\ and headache. After admission, he was diagnosed as a of \\ case right atrial (RA) myxoma by echocardiography. With \\ all preoperative preparation, he was undergone surgery
}

\section{Introduction:}

Primary cardiac tumor accounts for only $0.0017 \%$ to $0.19 \%$ of unselected patients at autopsy ${ }^{1}$. Seventyfive percent of these tumors are benign; $50 \%$ are myxomas ${ }^{2}$. Of myxomas, $75 \%$ to $80 \%$ are located on the left side of interatrial septum ${ }^{3}$. The reported rates in Bangladesh ${ }^{4}$ were $87.93 \%$ left atrial, $8.62 \%$ right atrial, $1.72 \%$ biatrial and $1.72 \%$ right ventricular myxoma. Prior to the development of cardiac catheterization in 1951, intracardiac tumors used to be diagnosed only at autopsy ${ }^{2}$. Since then echocardiography has replaced cardiac catheterization as the mainstay of diagnosis, because of its non-invasive advantages ${ }^{2}$.

The gross appearance of cardiac myxoma is variable ${ }^{5}$; however, they are generally polypoid,

a. Dr. M.A. Quashem, Professor of Cardiac Surgery, NICVD

b. Dr. Hriday Ranjan Roy, Resident, Cardiac Surgery, NICVD

c. Dr. Md. Golam Kibria, Assistant Professor, Cardiac Surgery, NICVD.

d. Dr. A. E. M. Mazharul Islam, Assistant Professor, Cardiology, DMCH.

e. Prof. A. A. Shafi Majumder, Professor Of Cardiology, NICVD.

f. Prof. N.A. Kamrul Ahsan, Professor Of Cardiac Surgery, NICVD.

g. Prof. N. U. Ahmed, Professor Of Cardiac Surgery, NICVD. National Institute of Cardiovascular Diseases and Hospital, Sher-E-Bangla Nagar, Dhaka.

Address of correspondence: Dr. Hriday Ranjan Roy, Resident, Cardiac Surgery, NICVD, Mobile- 01552-361096, Emailhriday_roy@yahoo.com

Received: 25 April, 2007

Accepted: 1 October, 2007 under general anesthesia with cardiopulmonary bypass (CPB). About $9 \mathrm{~cm} \times 7 \mathrm{~cm}$ myxoma was removed and severe tricuspid regurgitation (TR) was found by regurge test. De Vega annuloplasty of the tricuspid annulus was done. Recovery of the patient was satisfactory with disappearance of all symptoms.

(J Bangladesh Coll Phys Surg 2009; 27: 52-55)

mostly pedunculated, round or oval with a smooth surface, often covered with thrombus ${ }^{6}$. Cardiac myxomas are usually attached at the level of fossa ovalis of interatrial septum and located in left atrium ${ }^{7}$.

The first successful excision of left atrial myxoma was reported in $1951^{1}$. Right sided cardiac myxomas present to surgeons with a technical challenge because placement of the cannula for cardiopulmonary bypass can be difficult ${ }^{2}$.

Presented case demonstrates an atypically large $(9 \mathrm{~cm}$ $x 7 \mathrm{~cm}$ ) cardiac myxoma attached to the right atrial wall.

\section{Case Report}

A male of 45 years, cultivator, hailing from Cox's Bazar was admitted into NICVD on 21/11/2006 with the complaints of intermittent syncopal attack for 1 month, dyspnoea for $1 \frac{1}{2}$ months and low grade fever, malaise and headache for 2 months. The fever was irregular in nature and was not associated with cough or hemoptysis. His dyspnoea occurred mostly while he lies on bed, but was not relieved by sitting upright. It also occasionally aggravated by exertion. He had no history of asthma or tuberculosis. During last 1 month, he began to have syncopal attack 2 to 3 times a day. Occasionally, it was associated with convulsion, palpitation and tightness of chest. He had no history of diabetes mellitus, hypertension or smoking.

For these above complaints, he was admitted into a Medical College Hospital on 5/11/2006 and was 
treated by anti-epileptic drugs. But as the symptoms did not improve, he was referred to Dhaka Medical College Hospital on 14/11/2006. He was empirically diagnosed as a case of cerebral malaria and was treated as such. But as the symptoms did not improve, an echo was done and was diagnosed as a case of right atrial (RA) myxoma and was referred to NICVD on 21/11/2006. Examination finding on admission (in NICVD) were: Appearance- ill looking, confused, pulse- $100 / \mathrm{min}$, B.P- $100 / 70 \mathrm{mmHg}$, neck veinsengorged, diastolic murmur at tricuspid area, lungclear, no jaundice, edema, enlarged lymph node or clubbing. Per abdominal examination revealed normal. Reevaluation was done in Cardiology Unit- I on 23/11/2006. Chest X-ray and ECG were within normal limit. 2D Echo (Fig-1) shows a large mass in right atrium- protruding into right ventricle. Other baseline investigations were within normal limits. He was then submitted urgently to SU-III for emergency surgery.

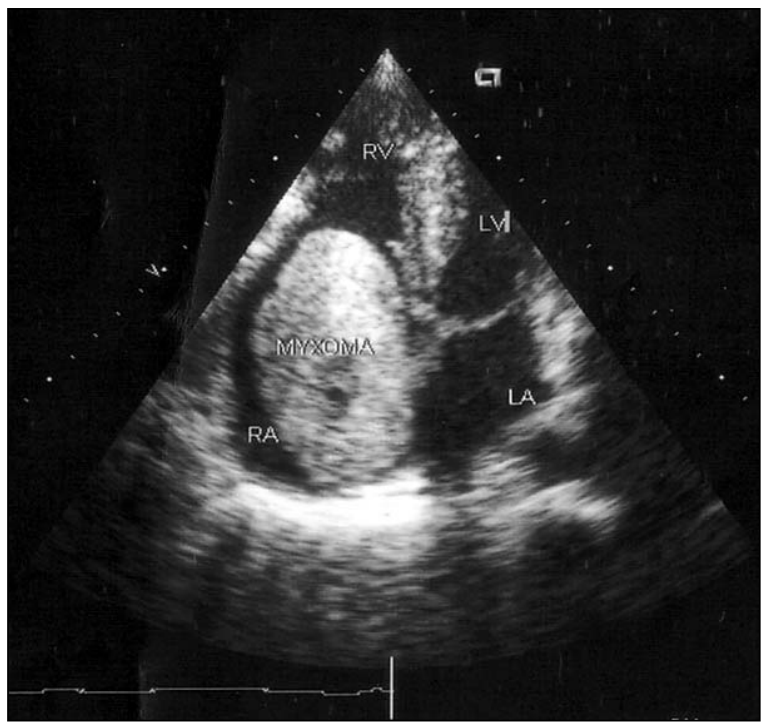

Fig.-1: 2D echocardiography

On 25/11/2006, under general anesthesia with cardiopulmonary bypass (CPB), open heart surgery was performed. CPB time was $96 \mathrm{~min}$ and cross clamp time was $52 \mathrm{~min}$. Per-operative findings and procedures were- A huge $(9 \mathrm{~cm} \times 7 \mathrm{~cm})$ encapsulated mass (Fig-2), occupying $2 / 3 \mathrm{rd}$ in RA and $1 / 3 \mathrm{rd}$ in right ventricle $(\mathrm{RV})$. It was attached to the limbus of fossa ovalis by a narrow stalk. Careful removal of the

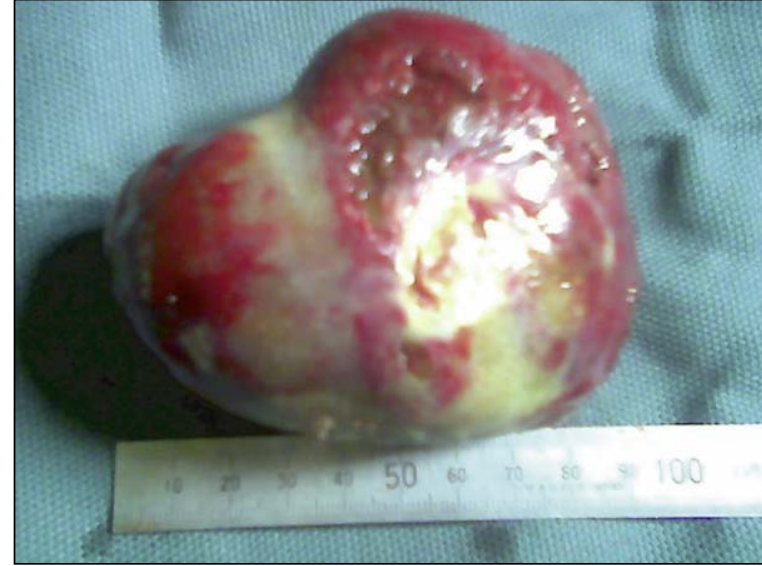

Fig.-2: The mass (myxoma) after removal.

mass was done to avoid pulmonary embolism with adequate tissue surrounding the stalk. RA \& RV cavity was washed with normal saline. The annulus of the tricuspid valve (TV) was found to be dilated. Peroperative valve regurge test showed severe tricuspid regurgitation (TR). De Vega annuloplasty of TV was done by $3 / 0$ prolene. Patient was kept under artificial ventilation and was extubated on the following morning. ICU outcome was smooth, but needed inotropic support with Dopa \& Dobuta for $2 / 3$ days. Histopathology report was consistent with myxoma (Fig-3). Post operative Echo showed TR grade- II, which was acceptable. Post operative pulmonary function was normal.

Finally, he was feeling well, all parameters were within normal limit and the neurological and constitutional symptoms disappeared.

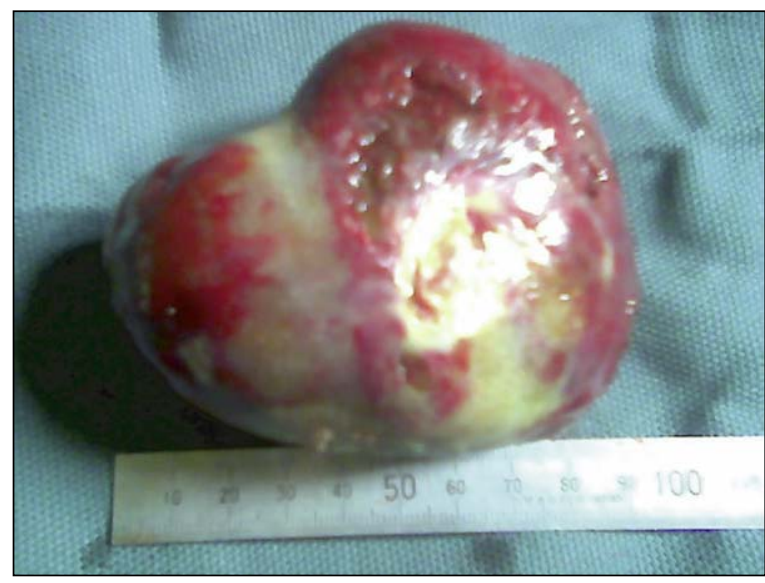

Fig.-3: Histopathology was consistent with myxoma. 


\section{Discussion:}

Right atrial myxoma accounts for only $15 \%$ to $20 \%$ of all cardiac myxomas ${ }^{2}$. It is usually found at the interatrial septum ${ }^{1}$, at the border of fossa ovalis. Atypical locations and multiple myxomas occur most frequently in case of familial myxoma ${ }^{8}$. The location also may be biatrial with atrial septal defect (ASD) ${ }^{9}$. Our patient's tumor was attached to the limbus of fossa ovalis by a narrow stalk in the right side of interatrial septum. This is a rare site of origination ${ }^{1}$, but family history was absent in presented patient.

Myxomas can present in any age group, but it occurs most often between the $3^{\text {rd }}$ and $6^{\text {th }}$ decade of life ${ }^{1}, 10$ as also in presented patient. In another report ${ }^{7}$, most of the sporadic myxomas are in females with mean age of 50 . In a report of Bangladesh $4,51.72 \%$ were male and $48.28 \%$ were female with patient's age ranged from 14 to 65 years. Presented case was a male of 45 years.

Myxomas are usually polypoid and pedunculated ${ }^{1}$. But in this patient, though it was pedunculated by a narrow stalk of about $1 \mathrm{~cm}$ width and $6 \mathrm{~mm}$ long, its surface was smooth, like an encapsulated mass with globular shape. In 2D echocardiographic evaluation, the presence of an attachment stalk allows to differentiate a myxoma from other masses ${ }^{7}$.

The size of the tumor varies. Rogland et $\mathrm{al}^{7}$ reported a myxoma sized $2 \mathrm{~cm}$ x $2 \mathrm{~cm}$; Gupta et al ${ }^{11}$ described $8 \mathrm{~cm}$ long tumor and Yazici et al 12 reported $6.3 \mathrm{~cm} \times 5.2 \mathrm{~cm}$ myxoma. Ours' was larger than all of those which was about $9 \mathrm{~cm} \times 7 \mathrm{~cm}$.

The tumor may have a considerable mobility at echocardiogram ${ }^{2}$, as also found in this case- which was even protruding into RVOT (right ventricular outflow tract) obstructing pulmonary circulations. The myxoma from left atrium (LA) may pass through ASD into RA and protrude into the TV producing Wrecking ball effect ${ }^{9}$. Mobile myxomas often exacerbate shortness of breath when patient assumes a particular posture ${ }^{13}$, which was also present in presented patient. The motion of the tumor can damage atrioventricular valve and rupture the cordae 1. In this case, the annulus of the TV was found to be hugely dilated with severe TR; which was repaired by De Vega annuloplasty.
Anemia and high ESR can be explained by continuous destruction of erythrocytes due to the "ball-valve" movement of the mass 1. But in presented patient, there was no anemia but had high ESR of $58 \mathrm{~mm}$ in $1^{\text {st }}$ hour (westergreen). The absence of anemia may be due to smoothness of the tumor.

Constitutional symptoms, including fever, malaise, weight loss and myalgia, are common in patients with myxoma and have been attributed to the findings that myxomas release cytokine interleukin-6, which is responsible for inflammatory and autoimmune manifestations ${ }^{13}$. All these were present in presented case patient, which disappeared after removal of the mass. Syncope is an extremely rare finding, and is likely due to intermittent complete occlusion of the tricuspid valve ${ }^{11}$. The syncopal attack was present in this patient \& disappeared after resection of the tumor.

There are reports of recurrence after excision, mostly in multiple, familial myxomas 2, 8, 14, 15. But as presented patient had no family history and it was single, the chance of recurrence is minimal. Quashem et al ${ }^{4}$ also found no recurrence in their series. In familial cases, Carney's complex, an autosomal dominant disease, there may be association of spotty skin pigmentation and endocrinopathies $6,8,16$. These symptoms were absent in this case, hence no chance to be a case of such.

The reported rate of left or right sided embolic phenomena associated with cardiac myxoma ranges from $21 \%$ to $33 \% 16,17$. In a report of NICVD ${ }^{4}$, overall $17.24 \%$ patients had clinical evidence of systemic embolism. In left sided myxomas, $21 \%$ to $33 \%$ may have systemic embolism and right sided myxoma have 2 to $24 \%$ chance of pulmonary embolism ${ }^{14,17}$. In presented patient, no evidence of pulmonary embolism was found and post operative pulmonary function was normal.

\section{Conclusion:}

Right atrial myxoma presents with a divergence of symptoms and make a diagnostic dilemma. An echocardiogram is invaluable to diagnose such a disease. Treatment should be done by immediate surgical removal- which will reduce morbidity and mortality. The disgraceful symptoms will also be dramatically disappeared after surgical treatment. 


\section{References:}

1. Reynen K. Cardiac myxomas. N Engl J Med. 1995; 333: 1610-1617.

2. Guhathakurta S and Riordan JP. Surgical treatment of right atrial myxoma. Tex Heart Inst J. 2000; 27: 61-63.

3. Imperio J, Summers D, Krasnow N, Piccone VA Jr. The distribution patterns of biatrial myxomas. Ann Thorac Surg. 1980; 29: 469-473.

4. Quashem MA, Shamsuddin A, Kibria G et al. Cardiac myxoma: Surgical treatment and follow up. A ten years experience in the National Institute of Cardiovascular Diseases, Dhaka. Bangladesh Heart J. 2006; 21(2): 78-80.

5. Araoz P, Mulvagh S, Tazelaar H, et al. CT and MR imaging of benign primary cardiac neoplasms with echocardiographic correlation. Radiographics. 2000; 20:1303-1319.

6. Hoffmann U, Globits S, Schima W, et al. Usefulness of magnetic resonance imaging of cardiac and paracardiac masses. Am J Cardiol. 2003; 92: 890-895.

7. Ragland MM and Tak T. Detection of right atrial myxoma by echocardiography. Cardiol in Rev. 2006; 14(2): 99-100.

8. McCarthy PM, Piehler JM, Schaff HV et al. The significance of multiple, recurrent, and "complex" cardiac myxomas. J Thorac Cardiovasc Surg. 1986; 91: 389-396.
9. Farid N and Gupta HP. Biatrial myxoma : a case report and the review of literature. Bangladesh Heart J. 2004; 19(1): 88-90.

10. Heath D. Pathology of cardiac tumors. Am J Cardiol. 1968; 21: 315-327.

11. Gupta D, Molina E, Palma J. Massive right atrial myxoma presented with syncope. Heart Vessel. 2006; 21: 256-257.

12. Yazici M, Ozhan H, Tetik O, Kinay O, Ergene O. Isolated large right atrial myxoma manifested by syncope. Text Heart Inst J. 2004; 31(2): 324-325.

13. Goodwin JF. The spectrum of cardiac tumors. Am J Cardiol. 1968; 21:307-314.

14. Parson AM and Detterbeck FC. Multifocal right atrial myxoma and pulmonary embolism. Ann Thorac Surg. 2003; 75: $1323-1324$

15. Bhan A, et al. Surgical experience with intracardiac myxomas: long-term follow-up. Ann Thor Surg. 1998; 66:810-813.

16. Mahilmaran A, Seshadri M, Nayar PG, Sudarsana G, Abraham KA. Familial cardiac myxoma: Carney's complex. Text Heart Inst J. 2003; 30(1): 80-81.

17. Bjessmo S, Ivert T. Cardiac myxoma: 40 years' experience in 63 patients. Ann Thorac Surg. 1997; 63:697-700. 